\title{
Psychiatric Rehabilitation Journal in the Era of COVID-19
}

Sandra G. Resnick, David Roe, Michelle P. Salyers

Sandra G. Resnick Northeast Program Evaluation Center, Office of Mental Health and Suicide Prevention, VA Central Office, Department of Veterans Affairs, West Haven, Connecticut, and Department of Psychiatry, Yale University School of Medicine

David Roe Department of Community Mental Health, Faculty of Social Welfare and Health Sciences, University of Haifa

Michelle P. SalyersDepartment of Psychology, Purdue School of Science, Indiana University-Purdue University Indianapolis

\begin{abstract}
The global health crisis caused by the coronavirus pandemic (COVID-19) has brought about previously unimaginable changes to all of health care, including the field of psychiatric rehabilitation. Given these dramatic changes, we pose this editorial to contemplate our mission and how we can best serve the field. We raise questions about how psychiatric rehabilitation will evolve and restate our steadfast commitment to publishing impactful research that shapes the field. We also remain hopeful, knowing psychiatric rehabilitation practitioners, researchers, and participants are pragmatic, persistent, and resilient, and will find opportunities for creativity and innovation even during this difficult time.
\end{abstract}

KEYWORDS: psychiatric rehabilitation, COVID-19, resilience

This is the author's manuscript of the article published in final edited form as:

Resnick, S. G., Roe, D., \& Salyers, M. P. (2020). Psychiatric Rehabilitation Journal in the era of COVID-19. Psychiatric Rehabilitation Journal, 43(2), 83-84. http://dx.doi.org/10.1037/prj0000434 
The mission of Psychiatric Rehabilitation Journal (PRJ) is to advance "the evidence for and understanding of psychosocial treatment and recovery of people with psychiatric disabilities, consistent with the principles and values of psychiatric rehabilitation and person-centered care." As we write this editorial in late April 2020, the global health crisis caused by the coronavirus pandemic (COVID-19) has brought about rapid and dramatic changes to all of health care including the field of psychiatric rehabilitation. So much about our world has changed almost overnight, leaving us to contemplate our mission and how we can best serve the field.

These are early days. We can only speculate about the long-term impact of COVID-19 on the practice of psychiatric rehabilitation. Common recovery goals such as trying to get or keep a job, attending school, connecting with friends, and resuming participation in previously enjoyed leisure activities are not easy to achieve during regular times despite the body of evidencebased practices supporting their attainment. COVID-19 has raised numerous new and challenging questions about achieving these goals in a postpandemic world. How will our practices and service delivery models need to be modified in this new era of global economic crisis and physical distancing, which could last for several years (Kissler, Tedijanto, Goldstein, Grad, \& Lipsitch, 2020)? How do we help individuals adjust their recovery goals within a context of global uncertainty? What does community integration and inclusion mean while physically distancing? How do individuals with psychiatric disabilities obtain or maintain valued social roles such as being a worker, when unemployment rates are at once-in-a-lifetime high levels, or being a student, when all learning has gone online, or being a family member when we are remote or always together? How has the sudden shift from face-to-face to telephone and video calls influenced the accessibility and experience of services, especially for those without the necessary technology or skills? How can family members support their loved ones while coping with their own stress generated by COVID-19? How do we ensure the health and well-being of front-line practitioners? These are only some of the questions we are thinking about.

We at PRJ are also thinking about the future of science and publishing. When so much is uncertain, we are doing our best to stay the course. We remain committed to continuing to publish impactful papers that shape the field and to maintain the high-quality peer review 
operations you have grown to expect. However, we are aware that life has changed for all of us. Research is changing. Your studies may be on pause due to a myriad of reasons, including restricted face-to-face interactions or loss of resources as your health systems focus only on supporting critical services. Your work may be shifting to respond to the crisis, perhaps with uncertainty about how services, operations, and research will continue. Given these unknowns, although we are committed to maintaining journal operations, we are building in flexibility. If you have received an invitation for peer review, you have noticed that we are offering additional time for those who review, understanding that circumstances may be unpredictable. If you are an author with an article under review, review times may be longer than usual to allow our reviewers the time they require to provide you with the expert constructive feedback you expect from PRJ. Similarly, if you are an author and require additional time for submitting a revision, as always, please contact our editorial office and we will gladly adjust the deadlines.

One of the lessons oft heard now is that the pandemic has reinforced the power of connection. The power of community to enact positive change is something that the psychiatric rehabilitation field has valued from our inception as a field. Psychiatric rehabilitation is by definition person-centered care, built on a foundation of connection. Another key feature of our field is that psychiatric rehabilitation practitioners, researchers, and participants are pragmatic, persistent, and resilient. Members of the psychiatric rehabilitation have long adjusted to difficult circumstances and innovated our way through. One bright side of this crisis may be new strengths we recognize in ourselves and each other, and an opportunity to connect with others on a deeper empathic level. For example, we may develop new understanding of what it is like to struggle with anxiety, hypervigilance, and social isolation. And the people who have long struggled with these experiences may feel less alone and possibly better understood. Clearly, "the times they are a-changin'" as a recent Nobel prize winner put it. While we cannot predict the future, we can predict one thing-we know that the creativity and dedication in this community will rise up and achieve what was before thought to be impossible. And we will be here to provide a scholarly voice for these milestones. If you have ideas about how the journal can support you during this time, please e-mail us. We look forward to your feedback. 


\section{Reference}

Kissler, S. M., Tedijanto, C., Goldstein, E., Grad, Y. H., \& Lipsitch, M. (2020). Projecting the transmission dynamics of SARS-CoV-2 through the postpandemic period. Science. Advance online publication. https://doi.org/10.1126/science.abb5793 